Start of body part 2University of Greifswald (May, 1994)

for double spacing

hep-th/9509079

PHYSICS LETTERS B, 336 (1994) 171-177

\title{
The heat kernel on symmetric spaces via integrating over the group of isometries
}

\author{
I. G. Avramidi $* \S$ \\ Department of Mathematics, University of Greifswald \\ Jahnstr. 15a, 17489 Greifswald, Germany \\ E-mail: avramidi@math-inf.uni-greifswald.d400.de
}

\begin{abstract}
A new algebraic approach for calculating the heat kernel for the Laplace operator on any Riemannian manifold with covariantly constant curvature is proposed. It is shown that the heat kernel operator can be obtained by an averaging over the Lie group of isometries. The heat kernel diagonal is obtained in form of an integral over the isotropy subgroup.
\end{abstract}

\footnotetext{
* Alexander von Humboldt Fellow

$\S$ On leave of absence from Research Institute for Physics, Rostov State University, Stachki 194, Rostov-on-Don 344104, Russia
} 


\section{Introduction}

The heat kernel $U(t)=\exp (t \square)$ associated with the Laplace-Beltrami operator acting on a $d$-dimensional Riemannian manifold $M$ without boundary with the metric $g_{\mu \nu}$ of Euclidean signature is of fundamental importance in mathematical physics [1-21]. In present paper we consider only the heat kernel diagonal

$$
\left.U(t)\right|_{\text {diag }}=\left.\exp (-t H) g^{-1 / 2} \delta\left(x, x^{\prime}\right)\right|_{x=x^{\prime}}
$$

and restrict ourselves to manifolds with covariantly constant curvature

$$
\nabla_{\mu} R_{\alpha \beta \gamma \delta}=0
$$

We are not going to investigate in this paper the effects of the topology but concentrate our attention on the local effects. We calculate the heat kernel diagonal rather formal, having in mind to get the asymptotic expansion at $t \rightarrow 0$ that does not depend on the global structure of the manifold at all. This will then mean that we have obtained all the terms without derivatives of the curvature in the asymptotic expansion of the heat kernel.

The condition (2) determines the geometry of the symmetric spaces [22]. The frame components of the curvature tensor (with respect to some frame $e_{\mu}^{a}$ that is parallel along geodesics) are constant and can be presented in the form

$$
R_{a b c d}=\beta_{i k} E_{a b}^{i} E_{c d}^{k}
$$

where $E_{a b}^{i},(i=1, \ldots, p ; p \leq d(d-1) / 2)$, is some set of antisymmetric matrices. The matrix $\beta_{i k}$ is known to be the metric of the isotropy algebra $\mathcal{H}$ with the generators $D_{i}=\left\{D^{a}{ }_{i b}\right\}$

$$
\begin{gathered}
D_{i b}^{a}=-\beta_{i k} E^{k}{ }_{c b} g^{c a}=-D_{b i}^{a}, \\
{\left[D_{i}, D_{k}\right]=F^{j}{ }_{i k} D_{j},}
\end{gathered}
$$

$F_{i k}^{j}$ being the structure constants.

It is not difficult to show that the condition of integrability of the equations (2) brings into existence a Lie algebra $\mathcal{G}$ of dimension $\operatorname{dim} \mathcal{G}=D=p+d$, with structure constants $C_{B C}^{A}=-C_{C B}^{A},(A=1, \ldots, D)$

$$
\begin{gathered}
C_{a b}^{i}=E_{a b}^{i}, \quad C_{i b}^{a}=D_{i b}^{a}, \quad C_{k l}^{i}=F_{k l}^{i}, \\
C_{b c}^{a}=C_{k a}^{i}=C_{i k}^{a}=0
\end{gathered}
$$

Introducing a symmetric nondegenerate matrix

$$
\gamma_{A B}=\left(\begin{array}{cc}
g_{a b} & 0 \\
0 & \beta_{i k}
\end{array}\right)
$$


that plays the role of the metric on the algebra $\mathcal{G}$ one can show that the adjoint and coadjoint representations of the algebra $\mathcal{G}$ are equivalent, i.e.

$$
\gamma_{A B} C_{C D}^{B}+\gamma_{D B} C_{C A}^{B}=0 .
$$

The generators of infinitesimal isometries $\xi_{A}=\left(P_{a}, L_{i}\right)$ of symmetric spaces can be presented in the form $[19,20]$

$$
\begin{aligned}
& P_{a}=P_{a}^{\mu} \nabla_{\mu}=-(\sqrt{K} \cot \sqrt{K})_{a}^{b} \mathcal{D}_{b}, \\
& L_{i}=L^{\mu}{ }_{i} \nabla_{\mu}=-D^{b}{ }_{i a} \sigma^{a} \mathcal{D}_{b},
\end{aligned}
$$

where $\sigma^{a}\left(x, x^{\prime}\right)$ are the frame components of the tangent vector to the geodesic between $x$ and $x^{\prime}, \mathcal{D}_{a}=\partial / \partial \sigma^{a}$ and $K=\left\{K_{b}^{a}\left(x, x^{\prime}\right)\right\}$ is a matrix defined by

$$
K_{b}^{a}=R_{c b d}^{a} \sigma^{c} \sigma^{d} .
$$

It is the algebra $\mathcal{G}$ that is generated by infinitesimal isometries [22]

$$
\left[\xi_{A}, \xi_{B}\right]=C_{A B}^{C} \xi_{C}
$$

\section{Heat kernel operator}

It is not difficult to show that the Laplacian in symmetric space can be presented in terms of generators of isometries

$$
\square=g^{\mu \nu} \nabla_{\mu} \nabla_{\nu}=\gamma^{A B} \xi_{A} \xi_{B}=g^{a b} P_{a} P_{b}+\beta^{i k} L_{i} L_{k},
$$

where $\gamma^{A B}=\left(\gamma_{A B}\right)^{-1}$ and $\beta^{i k}=\left(\beta_{i k}\right)^{-1}$.

Using this representation one can prove the following theorem [20].

\section{Theorem.}

For the compact Lie group $G$ (12) with positive definite metric $\gamma_{A B}(7)$ and the structure constants $C_{B C}^{A}$ satisfying the identity (8) it takes place the following identity for the Laplace operator (13)

$$
\begin{aligned}
\exp (t \square)= & (4 \pi t)^{-D / 2} \int d k \gamma^{1 / 2} \operatorname{det}\left(\frac{\sinh \left(k^{A} C_{A} / 2\right)}{k^{A} C_{A} / 2}\right)^{1 / 2} \\
& \times \exp \left\{-\frac{1}{4 t} k^{A} \gamma_{A B} k^{B}+\frac{1}{6} R_{G} t\right\} \exp \left(k^{A} \xi_{A}\right)
\end{aligned}
$$

or, equivalently,

$$
\begin{aligned}
\exp (t \square)= & (4 \pi t)^{-D / 2} \int d q d \omega \eta^{1 / 2} \beta^{1 / 2} \operatorname{det}\left(\frac{\sinh \left(\left(q^{a} C_{a}+\omega^{i} C_{i}\right) / 2\right)}{\left(q^{a} C_{a}+\omega^{i} C_{i}\right) / 2}\right)^{1 / 2} \\
& \times \exp \left\{-\frac{1}{4 t}\left(q^{a} g_{a b} q^{b}+\omega^{i} \beta_{i k} \omega^{k}\right)+\left(\frac{1}{8} R+\frac{1}{6} R_{H}\right) t\right\} \exp \left(q^{a} P_{a}+\omega^{i} L_{i}\right),
\end{aligned}
$$


where $\gamma=\operatorname{det} \gamma_{A B}, \beta=\operatorname{det} \beta_{i k}, \eta=\operatorname{det} g_{a b}, R_{G}$ is the scalar curvature of the group $G$

$$
R_{G}=-\frac{1}{4} \gamma^{A B} C_{A D}^{C} C_{B C}^{D}=\frac{3}{4} R+R_{H},
$$

and $R_{H}$ is the scalar curvature of the isotropy subgroup $H$

$$
R_{H}=-\frac{1}{4} \beta^{i k} F_{i l}^{m} F_{k m}^{l},
$$

with $\beta^{i k}=\left(\beta_{i k}\right)^{-1}$, the matrices $C_{A}=\left\{C^{B}{ }_{A C}\right\}=\left(C_{a}, C_{i}\right)$ are the generators of the adjoint representation of the algebra defined by

$$
C_{a}=\left(\begin{array}{cc}
0 & D^{b}{ }_{a i} \\
E^{j}{ }_{a c} & 0
\end{array}\right), \quad C_{i}=\left(\begin{array}{cc}
D^{b}{ }_{i a} & 0 \\
0 & F^{j}{ }_{i k}
\end{array}\right),
$$

and the integration is to be taken over the whole Euclidean space $\mathbb{R}^{\mathrm{D}}$.

Proof.

First one can show that

$$
\square \exp \left(k^{A} \xi_{A}\right)=X_{2} \exp \left(k^{A} \xi_{A}\right)
$$

where $X_{2}=\gamma^{B C} X_{B} X_{C}$ and $X_{A}=X_{A}^{M}(k) \partial / \partial k^{M}$ are the left-invariant vector fields on the group

$$
X_{A}^{M}(k)=\left(\frac{k^{A} C_{A}}{\exp \left(k^{A} C_{A}\right)-1}\right)_{A}^{M} .
$$

Then, introducing the metric on the group manifold

$$
G_{M N}=\gamma_{A B} X_{M}^{-1 A} X_{N}^{-1 B}
$$

and integrating by parts one has from (19)

$$
\begin{aligned}
& \square \int d k \gamma^{1 / 2} \Phi(t \mid k) \exp \left(k^{A} \xi_{A}\right) \\
& =\int d k \gamma^{1 / 2} \exp \left(k^{A} \xi_{A}\right)\left(G^{1 / 2} X_{2} G^{-1 / 2} \Phi(t \mid k)\right) .
\end{aligned}
$$

where $G=\operatorname{det} G_{M N}$ and

$$
\Phi(t \mid k)=(4 \pi t)^{-D / 2} \operatorname{det}\left(\frac{\sinh \left(k^{A} C_{A} / 2\right)}{k^{A} C_{A} / 2}\right)^{1 / 2} \exp \left\{-\frac{1}{4 t} k^{A} \gamma_{A B} k^{B}+\frac{1}{6} R_{G} t\right\} .
$$

Further, using the equations

$$
X_{2} G^{-1 / 4}=\frac{1}{6} R_{G} G^{-1 / 4}
$$

and

$$
k^{A} \frac{\partial}{\partial k^{A}} G^{-1 / 4}=\frac{1}{2}\left(D-X_{A}^{A}\right) G^{-1 / 4}
$$


that hold on the group manifold [21], one can show that $\Phi(t \mid k)$ satisfies the equation

$$
\partial_{t} \Phi=G^{1 / 2} X_{2} G^{-1 / 2} \Phi
$$

and initial condition

$$
\left.\Phi(t \mid k)\right|_{t=0}=\gamma^{-1 / 2} \delta(k)
$$

Therefrom it follows

$$
\left(\partial_{t}-\square\right) \int d k \gamma^{1 / 2} \Phi(t \mid k) \exp \left(k^{A} \xi_{A}\right)=0
$$

and hence

$$
\int d k \gamma^{1 / 2} \Phi(t \mid k) \exp \left(k^{A} \xi_{A}\right)=\exp (t \square)
$$

that proves the theorem.

\section{Heat kernel diagonal}

To get the heat kernel in coordinate representation one has to act with the heat kernel operator $\exp (t \square)(15)$ on the $\delta$-function (1). One can show that [20]

$$
\left.\exp \left(q^{a} P_{a}+\omega^{i} L_{i}\right) g^{-1 / 2} \delta\left(x, x^{\prime}\right)\right|_{x=x^{\prime}}=\left.\eta^{-1 / 2} \delta\left(\sigma_{0}^{a}(s, q, \omega)\right)\right|_{s=1}
$$

where $\sigma_{0}^{a}(s, q, \omega)$ is to be determined from the equation of characteristics

$$
\frac{d \sigma_{0}^{a}}{d s}=-\left(\sqrt{K\left(\sigma_{0}\right)} \cot \sqrt{K\left(\sigma_{0}\right)}\right)^{a}{ }_{b} q^{b}-\omega^{i} D^{a}{ }_{i b} \sigma_{0}^{b}
$$

with initial condition

$$
\left.\sigma_{0}^{a}\right|_{s=0}=0
$$

Solving the eq. (31) for small $\sigma_{0}^{a}$ we have

$$
\left.\sigma_{0}^{a}(s, q, \omega)\right|_{s=1}=\left(\frac{\exp \left(-s \omega^{i} D_{i}\right)-1}{\omega^{i} D_{i}}\right)_{b}^{a} q^{b}+O\left(q^{2}\right)
$$

and, therefore,

$$
\left.\delta\left(\sigma_{0}^{a}(s, q, \omega)\right)\right|_{s=1}=\operatorname{det}\left(\frac{\sinh \left(\omega^{i} D_{i} / 2\right)}{\omega^{i} D_{i} / 2}\right)^{-1} \delta(q) .
$$

Now substituting (30) and (34) in (15) we can easily integrate over $q$ to get finally the heat kernel diagonal

$$
\begin{aligned}
\left.U(t)\right|_{\text {diag }}= & (4 \pi t)^{-D / 2} \int d \omega \beta^{1 / 2} \operatorname{det}\left(\frac{\sinh \left(\omega^{i} F_{i} / 2\right)}{\omega^{i} F_{i} / 2}\right)^{1 / 2} \operatorname{det}\left(\frac{\sinh \left(\omega^{i} D_{i} / 2\right)}{\omega^{i} D_{i} / 2}\right)^{-1 / 2} \\
& \times \exp \left\{-\frac{1}{4 t} \omega^{i} \beta_{i k} \omega^{k}+\left(\frac{1}{8} R+\frac{1}{6} R_{H}\right) t\right\}
\end{aligned}
$$


where the matrices $F_{i}=\left\{F_{i j}^{k}\right\}$ are the generators of the isotropy algebra in the adjoint representation.

One can present this result also in an alternative nontrivial rather formal way. Substituting the equation

$$
(4 \pi t)^{-p / 2} \beta^{1 / 2} \exp \left(-\frac{1}{4 t} \omega^{i} \beta_{i k} \omega^{k}\right)=(2 \pi)^{-p} \int d p \exp \left(i p_{k} \omega^{k}-t p_{k} \beta^{k n} p_{n}\right)
$$

into the integral (35), integrating over $\omega$ and changing the integration variables $p_{k} \rightarrow$ $i t^{-1 / 2} p_{k}$ we get finally an expression without any integration

$$
\begin{aligned}
\left.U(t)\right|_{\text {diag }} & =(4 \pi t)^{-d / 2} \exp \left(t\left(\frac{1}{8} R+\frac{1}{6} R_{H}\right)\right) \\
& \times\left.\operatorname{det}\left(\frac{\sinh \left(\sqrt{t} \partial^{k} F_{k} / 2\right)}{\sqrt{t} \partial^{k} F_{k} / 2}\right)^{1 / 2} \operatorname{det}\left(\frac{\sinh \left(\sqrt{t} \partial^{k} D_{k} / 2\right)}{\sqrt{t} \partial^{k} D_{k} / 2}\right)^{-1 / 2} \exp \left(p_{n} \beta^{n k} p_{k}\right)\right|_{p=0} .
\end{aligned}
$$

where $\partial^{k}=\partial / \partial p_{k}$.

This formal solution should be understood as a power series in the derivatives $\partial^{i}$ that is well defined and determines the heat kernel asymptotic expansion at $t \rightarrow 0$.

\section{Concluding remarks}

In present paper we proposed a new purely algebraic approach for calculating the heat kernel diagonal in symmetric spaces that is based essentially on the Lie group of isometries.

We proved a theorem that expresses the heat kernel operator $\exp (t \square)$, i.e. the exponential of the second order operator, in terms of the isometries $\exp \left(k^{A} \xi_{A}\right)$, i.e. the exponential of first order operator.

Let us mention, that our formulae for the heat kernel diagonal are exact (up to possible nonanalytic topological contributions). This gives a nontrivial example how the heat kernel can be constructed using only the commutation relations of some differential operators, namely the generators of infinitesimal isometries of the symmetric space. These formulae can be used now to generate asymptotic expansion of the heat kernel for any symmetric space, any space with covariantly constant curvature, simply by expanding them in a power series in $t$.

In present paper we considered for simplicity the case of symmetric space of compact type, i.e. with positive curvature (positive definite matrix $\beta_{i k}$ ). There is a remarkable duality relation between the compact and noncompact symmetric spaces [22, 20] $R_{\alpha \beta \gamma \delta}^{*}=$ $-R_{\alpha \beta \gamma \delta}, \beta_{i k}^{*}=-\beta_{i k}, E_{a b}^{i *}=-E_{a b}^{i}, D_{i b}^{a *}=D_{i b}^{a}, F_{j k}^{i *}=F_{j k}^{i *}$. Therefore, one can get the results for noncompact case by means of analytic continuation. This means that our formulae (35) and (37) should be valid in general case of arbitrary symmetric space that is the product of compact, noncompact and the Euclidean ones. Moreover, It should also 
be valid for the case of pseudo-Euclidean signature of the metric $g_{\mu \nu}$. However, it is not perfectly clear how to do the analytic continuation for obtaining physical results.

\section{Acknowledgements}

I would like to thank G. A. Vilkovisky for many helpful discussions and R. Schimming and J. Eichhorn for their hospitality at the University of Greifswald. I am also grateful to P. B. Gilkey, H. Osborn, S. Fulling, T. Osborn, S. Odintsov and K. Kirsten for correspondence. This work was supported, in part, by a Soros Humanitarian Foundations Grant awarded by the American Physical Society and by an Award through the International Science Foundation's Emergency Grant competition.

\section{References}

[1] B. S. De Witt, Dynamical theory of groups and fields (Gordon and Breach, New York, 1965); in: Relativity, groups and topology II, ed. by B. S. De Witt and R. Stora (North Holland, Amsterdam, 1984) p. 393

[2] G. A. Vilkovisky, in: Quantum theory of gravity, ed. S. Christensen (Hilger, Bristol, 1983) p. 169

[3] A. O. Barvinsky and G. A. Vilkovisky, Phys. Rep. C 119 (1985) 1

[4] I. G. Avramidi, Nucl. Phys. B 355 (1991) 712

[5] P. B. Gilkey, Invariance theory, the heat equation and the Atiyah - Singer index theorem (Publish or Perish, Wilmington, DE, USA, 1984)

[6] J. Hadamard, Lectures on Cauchy's Problem, in: Linear Partial Differential Equations (Yale U. P., New Haven, 1923)

S. Minakshisundaram and A. Pleijel, Can. J. Math. 1 (1949) 242

R. T. Seeley, Proc. Symp. Pure Math. 10 (1967) 288

H. Widom, Bull. Sci. Math. 104 (1980) 19

R. Schimming, Beitr. Anal. 15 (1981) 77; Math. Nachr. 148 (1990) 145

[7] N. E. Hurt, Geometric quantization in action: applications of harmonic analysis in quantum statistical mechanics and quantum field theory, (D. Reidel Publishing Company, Dordrecht, Holland, 1983)

[8] P. B. Gilkey, Functorality and heat equation asymptotics, in: Colloquia Mathematica Societatis Janos Bolyai, 56. Differential Geometry, (Eger (Hungary), 1989), (NorthHolland, Amsterdam, 1992), p. 285

Forty More Years of Ramifications: Spectral Asymptotics and Its Applications, Ed. by S. A. Fulling and F. J. Narcowich, Discourses in Mathematics and Its Applications, No 1, Department of Mathematics, Texas A \& M University, College Station, Texas, 1991

R. Schimming, Calculation of the heat kernel coefficients, in: B. Riemann Memorial Volume, ed. T. M. Rassias, (World Scientific, Singapore), to be published

[9] P. B. Gilkey, J. Diff. Geom. 10 (1975) 601

[10] I. G. Avramidi, Teor. Mat. Fiz. 79 (1989) 219; Phys. Lett. B 238 (1990) 92 
[11] P. Amsterdamski, A. L. Berkin and D. J. O'Connor, Class. Quantum Grav. 6 (1989) 1981

[12] T. P. Branson and P. B. Gilkey, Comm. Part. Diff. Eq. 15 (1990) 245

N. Blazic, N. Bokan and P. B. Gilkey, Indian J. Pure Appl. Math. 23 (1992) 103

M. van den Berg and P. B. Gilkey, Heat content asymptotics of a Riemannian manifold with boundary, University of Oregon preprint (1992)

S. Desjardins and P. B. Gilkey, Heat content asymptotics for operators of Laplace type with Neumann boundary conditions, University of Oregon preprint (1992)

M. van den Berg, S. Desjardins and P. B. Gilkey, Functorality and heat content asymptotics for operators of Laplace type, University of Oregon preprint (1992)

[13] G. Cognola, L. Vanzo and S. Zerbini, Phys. Lett. B 241 (1990) 381

D. M. Mc Avity and H. Osborn, Class. Quantum Grav. 8 (1991) 603; Class. Quantum Grav. 8 (1991) 1445; Nucl. Phys. B 394 (1993) 728

A. Dettki and A. Wipf, Nucl. Phys. B 377 (1992) 252

I. G. Avramidi, Yad. Fiz. 56 (1993) 245

[14] S. A. Fulling, SIAM J. Math. Anal. 13 (1982) 891; J. Symb. Comput. 9 (1990) 73

S. A. Fulling and G. Kennedy, Trans. Am. Math. Soc. 310 (1988) 583

V. P. Gusynin, Phys. Lett. B 255 (1989) 233

[15] I. G. Avramidi, Yad. Fiz. 49 (1989) 1185; Phys. Lett. B 236 (1990) 443

[16] T. Branson, P. B. Gilkey and B. Ørsted, Proc. Amer. Math. Soc. 109 (1990) 437

[17] A. O. Barvinsky and G. A. Vilkovisky, Nucl. Phys. B 282 (1987) 163; Nucl. Phys. B 333 (1990) 471

G. A. Vilkovisky, Heat kernel: recontre entre physiciens et mathematiciens, preprint CERN-TH.6392/92 (1992), in: Proc. of Strasbourg Meeting between physicists and mathematicians (Publication de l' Institut de Recherche Mathématique Avancée, Université Louis Pasteur, R.C.P. 25, vol.43 (Strasbourg, 1992)), p. 203

A. O. Barvinsky, Yu. V. Gusev, V. V. Zhytnikov and G. A. Vilkovisky, Covariant perturbation theory (IY), Report of the University of Manitoba (University of Manitoba, Winnipeg, 1993)

[18] I. G. Avramidi, Phys. Lett. B 305 (1993) 27

[19] I. G. Avramidi, Covariant methods for calculating the low-energy effective action in quantum field theory and quantum gravity, University of Greifswald (1994), grqc/9403036

[20] I. G. Avramidi, A new algebraic approach for calculating the heat kernel in quantum gravity, University of Greifswald, EMA-MAT-1994-5, hep-th/9406047

[21] J. S. Dowker, Ann. Phys. (USA) 62 (1971) 361; J. Phys. A 3 (1970) 451

A. Anderson and R. Camporesi, Commun. Math. Phys. 130 (1990) 61

R. Camporesi, Phys. Rep. 196 (1990) 1

[22] H. S. Ruse, A. G. Walker, T. J. Willmore, Harmonic spaces, (Edizioni Cremonese, Roma (1961))

J. A. Wolf, Spaces of constant curvature (University of California, Berkeley, CA, 1972)

B. F. Dubrovin, A. T. Fomenko and S. P. Novikov, The Modern Geometry: Methods and Applications (Springer, N.Y. 1992) 